\title{
DESEOS, CREENCIAS Y OPORTUNIDADES EN LA TOMA DE DECISIONES DE ESTUDIANTES SECUNDARIOS EN TRÁNSITO A LAUNIVERSIDAD. ACCIONES Y REACCIONES EN TORNO A LA IMPLEMENTACIÓN DEL PUNTAJE RANKING ${ }^{12}$
}

\section{Nelson Paulus ${ }^{3}$, Christian Blanco $^{4}$}

\section{RESUMEN}

A partir de la implementación inicial del puntaje ranking de enseñanza media en los procesos del Sistema Único de Admisión (SUA) de Consejo de Rectores (CRUCH) entre 2013 y 2014, el estudio aborda la forma en que se configuró la migración de estudiantes de colegios públicos de alta exigencia a otros de distinto perfil. El trabajo reflexiona sobre la dimensión interactiva que subyace a la implementación de las políticas públicas, remitiéndonos a la experiencia de los estudiantes que, en tránsito de la educación secundaria a la superior, se transforman en actores que toman decisiones en el marco de un nuevo escenario regulativo. Por medio de entrevistas a directivos y estudiantes enfrentados a esa coyuntura se desarrolla un caso crítico, en el cual se analiza en lo esencial la experiencia de estos últimos a partir de sus deseos, sus creencias y oportunidades, utilizando el modelo DBO (Desires, Beliefs $\mathcal{E}$ Opportunities) de Hedström. Tras el análisis correspondiente, se observa que el modelo rinde en términos de su capacidad ilustrativa. No obstante, no agota el fenómeno correspondiente, por lo cual se proyectan nuevos elementos que necesariamente deben ser abordados para realizar un análisis apropiado.

Conceptos clave: tránsito a la educación superior, elección escolar, modelo $\mathrm{DBO}$, toma de decisiones, implementación de políticas públicas, ranking, sistema de admisión

1 Proyecto apoyado financieramente por CNED/Convocatoria 2017.

2 Los autores agradecen a la Universidad de Santiago de Chile, ya que esta investigación se enmarca en el proyecto DICYT - USACH Regular N 031652 PS “¿Quién se robó mi ranking? Motivos micro y efectos macro en la implementación del ranking de notas en los procesos de admisión a las universidades del CRUCH".

3 Universidad de Santiago de Chile, Santiago, Chile. Contacto: nelson.paulus@usach.cl

4 Instituto de Filosofía y Ciencias de la Complejidad, Santiago, Chile. Contacto: chblancoj@ gmail.com 


\title{
DESIRES, BELIEFS AND OPPORTUNITIES IN DECISION-MAKING OF SECONDARY STUDENTS HEADING TO UNIVERSITY. ACTIONS AND REACTIONS REGARDING THE IMPLEMENTATION OF THE RANKING SCORE IN THE ADMISSION SYSTEM
}

\begin{abstract}
This study addresses school choice and migration processes of students from academically demanding public schools, in comparison to students from schools with a different profile, in the context of the recently implemented high-school ranking score in the Single Admission System (SUA) of the Council of Rectors (CRUCH), between 2013 and 2014. The work addresses the interactive dimension that underlies the implementation of public policies, referring to the experience of students who, in the transition from secondary to higher education, become actors who make decisions within the framework of a new regulatory scenario. Through interviews with school leaders and students at this juncture, the research approaches a critical case in which student experience is analysed based on their desires, beliefs and opportunities, by means of the Hedström's DBO model (Desires, Beliefs \& Opportunities). The analysis showed that the model performs in terms of its illustrative capacity. However, it is not exhaustive regarding the phenomenon, as new elements emerged that need to be addressed to perform an appropriate analysis.
\end{abstract}

Key concepts: Transition to higher education, School choice, DBO model, Decision-making, Public Policy Implementation, Ranking, Admission System. 


\section{Introducción}

En las últimas décadas en Chile, el tránsito de la educación secundaria a la universitaria tradicional ${ }^{5}$ (constituida en términos generales por la oferta realizada por las universidades del Consejo de Rectores de Universidades Chilenas, $\mathrm{CRUCH}^{6}$ ) ha estado mediado por un sistema centralizado, que construye un puntaje a partir del cual se selecciona a los estudiantes. Este puntaje es requerido por las diversas instituciones y para las distintas carreras, considerando principalmente promedio de notas de enseñanza secundaria (Notas de Enseñanza Media, $\mathrm{NEM}^{7}$ ) y puntajes obtenidos en la Prueba de Selección Universitaria $\left(\mathrm{PSU}^{8}\right)$, administrada por Sistema Único de Admisión (SUA) del CRUCH e implementado través del DEMRE9 . En torno a este puntaje, las distintas universidades pertenecientes al CRUCH (y otras universidades privadas adscritas al sistema) estructuran su sistema de admisión conforme al cual seleccionan los estudiantes que cubrirán su oferta de matrícula ${ }^{10}$.

Las ponderaciones asignadas históricamente a cada uno de estos elementos han variado de universidad en universidad y, lógicamente, en los distintos programas de estudio dependiendo de su naturaleza específica, en tanto estos requerían y ponderaban de

5 En este trabajo nos referiremos esencialmente a la educación universitaria, entendida como la ofrecida por instituciones universitarias, en tanto supone en general un sistema de selección distinto al de otras instituciones de educación superior que funcionan en el sistema chileno, tales como institutos profesionales y centros de formación técnica. En este sentido, y dado que la dinámica de acceso a los programas ofrecidos por estos dos últimos tipos de instituciones no se encuentra afecto a una dinámica selectiva en términos académicos, a pesar de ser parte de la educación superior no forman parte del presente análisis.

6 Institución integrada por los rectores de las veintisiete universidades estatales y no estatales con vocación pública del país (pertenecientes a la iglesia católica y a antiguas fundaciones privadas) presidida por el ministro de Educación. Como institución, fue creada por ley el año 1954 y está constituida como una persona jurídica de derecho público y de administración autónoma, y su quehacer se orienta a la coordinación de la labor universitaria y al mejoramiento del rendimiento y calidad de su enseñanza (ver http://www.consejoderectores. cl/historia, acceso del 16-12-2017).

7 Promedio de las calificaciones de educación secundaria para Chile.

8 Prueba de conocimiento estandarizada, de administración centralizada y que se aplica una vez al año en el país.

9 Departamento de Evaluación, Medición y Registro Curricular, organismo colaborador del CRUCH.

10 Cabe destacar que, en los últimos años, algunas universidades privadas no tradicionales y así no pertenecientes al CRUCH se han sumado a este sistema de selección. 
manera diferenciada los puntajes obtenidos en las cuatro pruebas de la PSU. A saber: Lenguaje y Comunicación, Matemática, Ciencias (divididas a su vez en Biología, Física y Química) e Historia, Geografía y Ciencias Sociales.

En este contexto, la modificación más importante a este sistema observada en los últimos años es, sin duda, la incorporación y el posterior fortalecimiento de un nuevo ítem en la construcción de los puntajes de postulación, y que se asocia al puntaje de ranking. Respecto de este último, el año 2012, en sesión extraordinaria del 14 de junio (por medio del Acuerdo No 24/2012; ver Acta) ${ }^{11}$, el CRUCH lo incluyó entre los factores de selección para el ingreso a todas sus universidades, con el fin declarado de incrementar la inclusión sin perjudicar la excelencia académica.

El Ranking de Notas (en adelante el ranking), en tanto indicador, fue definido originalmente como una medida de la posición relativa que ocupa el estudiante respecto de la situación general del establecimiento educacional del cual egresa finalizando su educación secundaria ( $4^{\circ}$ de Enseñanza Media ${ }^{12}$ ). En su construcción - conforme a lo definido por el CRUCH - intentaba neutralizar variables que generaban inequidad en el acceso, tales como el tipo de establecimiento donde cursó sus estudios secundarios ${ }^{13}$ y su situación socioeconómica familiar. Para el primer año de introducción de este indicador (proceso de admisión 2013) y en el marco del pilotaje inicial, se determinó que tuviese una ponderación única y fija de un $10 \%$ para las postulaciones a realizar en todas las universidades partícipes del mismo sistema de admisión, dejando al arbitrio de las instituciones la forma en la cual la modificación de los otros puntajes, tradicionalmente considerados en el marco del proceso de admisión, se ajustaba para dar cabida a este nuevo ítem (Larrocau, Ríos y Mizala, 2013), estableciéndose también un máximo de 150 puntos máximo como bonificación del ranking por sobre las notas $\mathrm{NEM}^{14}$.

11 Ver http://www.consejoderectores.cl/public/pdf/acta/ACTA_SESION_ EXTRAORDINARIA_n_1_2012_jueves_14.06.2012.pdf

12 Curso con el cual se egresa de la educación secundaria en Chile.

13 En Chile: particular pagado, particular subvencionado o municipal (dependencia exclusivamente pública).

14 Ver http://www.consejoderectores.cl/public/pdf/acta/acta-2012/ACTA_SESION_535.pdf 
Aunque estas eran las condiciones de inicio, la idea contemplaba para años posteriores un progresivo aumento del peso específico de la ponderación del ranking en la construcción del puntaje final, por lo que comenzó a ser incrementado para la admisión a sus carreras por las distintas universidades; incluso, en algunos programas e instituciones universitarias, el peso específico de la ponderación del ranking en la construcción del puntaje final fue aumentado hasta un $40 \%$.

Sobre el particular, y más allá de las interrogantes referidas a si este nuevo indicador constituiría un mejor predictor del éxito académico en la educación universitaria, entre los distintos actores del sistema parecía haber consenso en que esta medida constituía un aporte para mejorar la equidad en el acceso a la educación universitaria de las distintas clases sociales ${ }^{15}$. No obstante, algunos autores (entre otros Gil, Paredes y Sánchez, 2013) expresaban preocupación respecto de que, dependiendo de los mecanismos específicos a partir de los cuales se implementase efectivamente, el ranking podría redundar en una nociva competencia entre los propios estudiantes: “...considerar la posición relativa del alumno en el curso o su generación, podría inducir a una competencia que, desde la perspectiva de las decisiones públicas, no se quiere introducir en el nivel escolar" (Gil, Paredes y Sánchez, 2013, p. 12).

Al respecto, la incorporación del puntaje ranking y su posterior fortalecimiento parece haber incidido en que muchos estudiantes pertenecientes a establecimientos secundarios de los así llamados "emblemáticos" (distinguibles por su carácter público, tradicional y de alta exigencia académica ${ }^{16}$ ) migraran atropelladamente hacia otros establecimientos educacionales sin ese perfil, situación que, en un primer momento, tensionó la intención original de la iniciativa pública, pues si bien pretendía corregir ciertas dinámicas de inequidad

15 En tanto podía reducir el efecto regresivo de utilizar casi exclusivamente en la construcción de puntajes ponderados para participar del proceso de admisión la prueba estandarizada PSU.

16 Este perfil se ha traducido regularmente en que quienes han egresado de establecimientos emblemáticos han tenido muy buenas oportunidades de ingresar a las universidades tradicionales del CRUCH. 
en la operación del sistema regular de admisión a las universidades tradicionales, encontró, en virtud de dichas migraciones, resistencias para su implementación.

En el marco del contexto aquí descrito, el presente estudio pretende abordar cuál fue la dinámica interactiva desarrollada por un conjunto de estudiantes que detentaban un background educativo, acumulado durante el tiempo, que en la situación original (previa a la incorporación del puntaje del ranking) les permitía proyectar de manera optimista su inserción en la educación universitaria de mayor selectividad (universidades tradicionales) y que en la situación posterior a la señalada incorporación, y en tanto "rankeados" en cierta posición específica (podríamos pensar de manera gruesa y preliminar, bajo la medianía de la tabla) ven modificadas sus expectativas de obtener los puntajes correspondientes que les permitiesen avanzar con sus planes originales de incorporación a la educación universitaria tradicional. Al parecer, muchos de estos estudiantes —a través de un cambio de colegio- pretendieron activar los mecanismos que les permitieran quedar en posiciones más avanzadas, si no de punta, en la distribución del ranking, de manera tal que esta maniobra (al menos aparentemente y considerando ceteris paribus la construcción de sus otros puntajes) les permitiría amortiguar el impacto de la incoporación del ranking en la construcción de sus puntajes, e incluso, en algunos casos, mejorarlo ${ }^{17}$.

Posterior a esta introducción, el marco conceptual aborda dos puntos centrales en la fundamentación del trabajo: el primero nos remite a la implementación de políticas públicas en el ámbito de la educación superior y la relevancia que tienen en ella las dinámicas interactivas, en tanto uno de los supuestos básicos que orienta el desarrollo de este trabajo consiste en entender que las políticas de educación superior prueban su éxito no solo en los macroprocesos

17 En un primer momento, en 2013 y 2014, el ranking se calculaba en función de la posición relativa del promedio NEM respecto de la generación de egreso. Posterior a esos años y como necesaria corrección en virtud de los fenómenos observados, dicho criterio se neutralizó, considerando como el valor del ranking el desempeño a lo largo de la enseñanza media en los distintos establecimientos donde esta haya sido cursada, circunstancia en la cual los estímulos para migrar de un colegio a otro se diluyen. 
políticos que lo deciden, sino que, de manera especial, en la forma en la cual estas iniciativas son implementadas y, en lo específico, recibidas por los destinatarios de esta (Trowler, 2002). En este sentido, cabe interrogarse por la forma en la que se configuró, en el marco de su implementación, el fenómeno de cambio de colegio que se observó, por parte de muchos estudiantes, desde colegios emblemáticos a otros de perfil distinto, y que fue reportado por la prensa de la época ${ }^{18}$. Responder esta interrogante resulta importante desde la óptica de las políticas públicas en general, en tanto interroga por la manera en la que, en su momento, se configuró una situación problemática para la intención original de la política específica. En la actualidad se puede observar que la política asociada a la implementación del ranking se encuentra consolidada, entre otras cosas, en virtud de los oportunos ajustes realizados con motivo de la situación en parte aquí descrita. No obstante lo anterior, y más allá de la situación específica, también existe en torno al caso un fuerte interés desde lo teórico, porque constituyó una excelente oportunidad para testear algunas presunciones implícitas en ciertos desarrollos asociados a la sociología y al aporte que las ciencias sociales pueden hacer a los procesos de implementación de las políticas públicas. Dicho componente teórico es abordado en la segunda parte del marco conceptual, mediante una breve contextualización que explica la utilización del modelo DBO para el análisis del caso en cuestión.

Posteriormente, la reseña metodológica, además de los detalles de la recolección de la información, caracteriza la pertinencia del estudio realizado como un caso crítico, el cual merece ser abordado en tanto contiene elementos de una situación excepcional generada en el devenir de la política pública. A continuación, y estructurado en torno a la secuencia $\mathrm{DBO}$, procede la revisión y el análisis del material recolectado, y la interpretación de este. Aunque se desarrolla primeramente en función de los elementos básicos contenidos en el modelo, es complementado con algunos elementos afines a la teoría de toma de decisiones y con algunos tópicos que emergen de la

18 Ver, por ejemplo, http://www.piees.cl/wp-content/uploads/2017/03/informe_ranking_2014. pdf visitado el 25-02-2019 
revisión del material correspondiente. Para finalizar, en la conclusión se aborda lo visto del caso general a partir del material revisado, y se contrasta con la visión de otros actores y observadores del mismo proceso, proyectando el análisis desarrollado a otros tópicos relevantes en los procesos de toma de decisiones, a propósito de la implementación de políticas públicas.

\section{Marco conceptual}

a) Implementación de políticas públicas en educación y dinámicas interactivas

Cabe destacar que el presente estudio nos remite circunstancialmente a la formulación de nuevas políticas en términos de admisión y acceso a la educación universitaria enunciadas por el CRUCH, que impactan en la configuración de las oportunidades que los estudiantes y sus familias tienen, de que estos puedan ingresar en la educación superior usualmente más cotizada en Chile, como es la universitaria pública y tradicional.

No obstante, en este punto cabe destacar la distinción realizada por Scharpf (1997), conforme a la cual, en el ámbito de la investigación en políticas públicas, uno debiese distinguir dos orientaciones: por una parte aquella referida a los sustantivo de los problemas específicos (Problem Oriented Policy Research: ¿cuál es el problema?, ¿qué pasa?, $y$, consecuentemente, ¿qué hacer?) y otra dirigida a la dimensión interactiva de éstas (Interaction Oriented Policy Research), que pone su atención en aquellos aspectos del procesamiento de las políticas públicas que derivan de la interacción entre diversos actores, tanto para la construcción como para la recepción e implementación de las correspondientes iniciativas, y que regularmente suelen constituir elementos imprevistos y obstáculos para la adecuada concreción de la política. En lo efectivo, y más allá del caso específico, el presente trabajo nos remite principalmente a dinámicas de tipo interactivo por parte de quienes reciben la iniciativa pública, en tanto asume que las políticas juegan su éxito no solo en los macroprocesos políticos, sino particularmente en los microprocesos que se desarrollan cotidianamente en la población que los recibe y que, en muchas 
oportunidades, a nivel agregado, configuran escenarios imprevistos para la intención original de la iniciativa pública ${ }^{19}$.

En materia de implementación de políticas públicas en educación superior, en el último tiempo ha cobrado relevancia la aproximación que a ellas se realiza desde una perspectiva que realza la complejidad que impone la conducción de la política pública en diversos niveles ${ }^{20}$. El interés en la implementación de las políticas públicas desde esta perspectiva implica que, al interior de los diferentes niveles a través de los cuales estas se procesan (nacional, regional, local, etc.) (Witte, 2003), resulta de especial interés responder a interrogantes referidas a cómo se adopta, cómo se adapta, cómo se interactúa y cómo se disputa con otros agentes en el marco de este desarrollo conjunto (Enders, Jeliazkova, McGuiness y Massen, 2003), destacando desde esta aproximación la importancia que en dicho procesamiento poseen los contextos que proveen los diversos niveles y al interior de los cuales los promotores y destinatarios de la política interactúan. Al respecto y en general, se estima que el traspaso y los consecuentes procesos de implementación y aplicación de las políticas abren diversas posibilidades para que los actores, ubicados en los distintos niveles (particularmente los de los niveles inferiores), puedan influenciar e incidir en aquello que viene "dado y enmarcado" desde los niveles superiores. Algo similar a lo que observaba Bernstein ${ }^{21}$ (1993), al señalar que cada ámbito social recontextualiza las prácticas sociales que recibe conforme a principios que le resultan específicos y que se desprenden de la particular forma de producción social asociada al contexto receptor, originándose de esta forma prácticas y discursos distintos del original.

Así, se estima que las grandes líneas de orientación y las iniciativas impulsadas por las declaraciones de intenciones quedan

19 En el ámbito específico de las políticas referidas al tránsito postsecundario, en Chile solo desde hace algunos años se ha podido observar algunas iniciativas que han abordado, por medio de métodos mixtos, tanto la perspectiva de actores, como los contextos estructurales vinculados a la toma de decisiones asociada al tránsito postsecundario (Paulus, Geoffroy y Mendoza, 2010; Treviño, Scheele y Flores, 2014; Leyton 2015; Leyton, Vásquez y Fuenzalida, 2012).

20 Multilevel governance (Witte, 2003).

21 Asociado a que, según Bernstein, el cambio en el discurso (1993) redunda en el cambio de las prácticas. Ver además Troiano (1999). 
predispuestas a ser reinterpretadas, ya no por las altas esferas de decisión sino por los actores de los ámbitos cotidianos en los que éstas se implementan. Conforme a lo señalado por Trowler (2001), una perspectiva más referida a los agentes, que aborde la política como texto, acentúa el rol de los actores en el proceso político, incluyendo su habilidad para discutir, negociar y reconstruir tanto la política misma como el discurso en el cual está codificada. Lo importante en este punto radica en reconocer la existencia de diversos niveles contextuales e institucionales en los cuales se toman decisiones respecto de la adopción e implementación de políticas públicas. Así visto, aquello que resulte de la implementación de la política será contingente, adquiriendo diversas formas en distintos contextos institucionales (Trowler, 2000).

Siendo el horizonte virtual que la adopción e implementación de una determinada política se desarrolle de manera armónica e integrada, es innegable la necesidad de que los actores situados en los diversos niveles acuerden formas consensuadas de asistencia y coordinación. Este panorama ofrece múltiples oportunidades de interacción y colaboración, pero también abre la posibilidad de diversas instancias de descoordinación y conflicto, pues en cada uno de estos niveles el procesamiento de las políticas se desarrolla conforme a su propio contexto e institucionalidad. La diversa situación de los contextos en los cuales se implementan las políticas tiene la desafortunada consecuencia (al menos desde la perspectiva de los responsables de las políticas públicas) de que estas tendrán diferentes resultados en los diversos ámbitos locales, donde es probable que se observen distintas configuraciones de fuerzas y factores contingentes que conducirán la recepción y la respuesta a la correspondiente política (Trowler, 2002). Conforme a esto, para efectos de comprender el procesamiento de las políticas debiese examinarse no solo los motivos, intereses y percepciones implicadas en una política específica, sino además introducirse en la forma en la cual esta es redefinida y reenmarcada por los distintos actores en las diversas etapas del proceso (Enders, Jeliazkova, McGuiness y Massen, 2003; Sabatier, 2005). 
Para dicho fin, se necesita un marco conceptual que permita abordar distintos elementos que subyacen a dichos procesos interactivos. Una importante alternativa para ello radica en las teorías de las ciencias sociales de extracción más sociológica, que nos remiten a la explicación de las acciones de los individuos considerando de manera especial sus contextos sociales e institucionales. Creemos que utilizando el modelo DBO (Desires, Beliefs and Opportunities) de Hedström (2005) dicho objetivo puede ser logrado, en tanto incorpora algunos complementos extraídos del Institucionalismo Centrado en Actores (ICA) (Scharpf, 1997), porque este tipo de teorías (centradas en actores) emergen en respuesta a los modelos top-down de las políticas públicas, considerando las preocupaciones, objetivos y tácticas de los actores para lidiar con la nueva política, y reconociendo la disparidad entre la política pública formal y la práctica (Sin, 2014). Lo anterior resulta particularmente relevante si estimamos que las políticas normalmente no instruyen a sus destinatarios respecto de lo que deben hacer, sino que crean circunstancias en las cuales el rango de opciones disponibles para decidir qué hacer se estrechan o cambian (Ball, 1994; Braun et al., 2011).

\section{b) Deseos, Creencias y Oportunidades}

En el ámbito de la toma decisiones en torno a opciones educativas, Gambetta (1987) contrapone a la noción de que los sujetos son esencialmente pasivos y así se encuentran constreñidos por la escasez de alternativas estructurales, la idea de que, si bien los individuos no son enteramente libres de elegir, sí son capaces de desarrollar acciones orientadas a propósitos, conforme a las cuales las decisiones en materia educativa pueden ser estimadas como acciones que ponderan alternativas en función de recompensas futuras. De alguna forma, temáticas similares ya han sido tratadas anteriormente por autores como Coleman (1966, a través del famoso Coleman Report), Kain \& Singleton (1996), Gamoran \& Long (2006) o Boudon (1974). Este último analizó la temática de la educación y la toma de decisiones asociada a ella a través de estrategias de simulación de la interacción entre la inequidad derivada de la diversa distribución de las oportunidades educacionales y los mecanismos de movilidad social (Elster, 1976). 
En el contexto de la presente investigación, intentamos abordar diversos componentes que contextualizan los procesos de decisión asociados al ámbito educativo como lo hace Gambetta (1987), tales como elementos subjetivos e interaccionales (Manzo, 2011; Manzo, 2013). En este sentido, la comparación que subyace en la obra de Gambetta entre restricciones y posibilidades (estructuras y decisiones) es muy ilustrativa de las propuestas teóricas de la sociología analítica contenidas en Aguiar, De Francisco y Noguera (2009), Barbera (2006), Goldthorpe (1998), Hedström \& Bearman (2011), Hedström \& Udehn (2011) y Noguera (2003 y 2006)22.

Respecto de estas últimas, y en lo relacionado a acciones y decisiones, para complementar la aproximación neoinstitucionalista provista por el ICA (Scharpf, 1997) y que nos remite al componente de naturaleza más estructural de la política pública, el presente estudio intenta poner en juego algunos de los contenidos del modelo de inspiración accionalista DBO (Desires, Beliefs \& Opportunities), propuesto por Hedström en su descripción de los principios de sociología analítica y contenidos en su obra del año 2005 (Dissecting the Social), revisados también por Bornmann (2010) y Kaidesoja (2012). De manera sucinta, el modelo DBO intenta explicar la forma en la que los deseos y las creencias, asociados a estructuras de oportunidades, son capaces de traducirse en acciones, dentro de las cuales también deben contarse las decisiones de los individuos. De alguna manera, una aproximación de esta naturaleza nos remite a una opción que nos permite

... ver la teoría social como la elaboración de modelos de acción basados en el estudio de las motivaciones, razones o preferencias de los individuos, así como de los efectos agregados, concatenaciones o composiciones de esas acciones individuales, efectos que no necesariamente son buscados o queridos por esos mismos individuos. (Noguera, 2003, p. 102)

22 Para una revisión crítica de las propuestas de la Sociología Analítica, ver Manzo (2010). 
El modelo DBO postula en lo esencial que, para entender aquello que resulta como una acción ${ }^{23}$, uno debe tener en mente los deseos, las creencias y las oportunidades de los individuos, así como los elementos regulativos, normativos y cognitivos (Scott, 1995) que aporta el contexto institucional en el que se desempeñan los actores (desarrollado en el ICA de Scharpf, 1997).

\section{Metodología}

El caso específico que se aborda nos remite a las interacciones desarrolladas por los destinatarios de la política (principalmente estudiantes) en el marco del proceso de implementación del puntaje ranking en el sistema de admisión a la educación universitaria tradicional chilena entre 2013 y 2014. El referido caso posee valor en términos de lo teórico, en tanto constituye lo que algunos metodólogos señalan e indican como un "caso crítico" (Yin, 2017); vale decir, una situación excepcional, fuera del control de los investigadores, acotada en el tiempo y en un contexto determinado, porque alude a estudiantes de colegios muy específicos.

Si bien la especificidad del caso cuestiona su generalización, abordar la situación específica de esa coyuntura y en dichos contextos institucionales posee especial valor, en tanto constituye una suerte de experimento natural (Dunning, 2012; Babbie, 2013) del cual los investigadores, sin haber intervenido en forma alguna en él, se sirven a posteriori y aprovechando la oportunidad para efectos de testear algunas teorías sociológicas. Si bien este tipo de diseño no está exento de problemas, derivados principalmente de su carácter solo cuasiexperimental al momento de comparar quién decide cambiarse de colegio y quién no, en virtud de ciertos ajustes puede ofrecer ricos elementos a considerar para el desarrollo teórico desde lo empírico.

Así visto, la estrategia general de la investigación es la de desarrollar un estudio de caso crítico, principalmente referido a

23 En este especialísimo caso a una decisión que nos remite a una suerte de School Choice, en la cual la alternativa básica es la de permanecer o no en el colegio de origen, considerando las implicancias que esto posea en términos de la oportunidad de obtener la cotizada educación universitaria tradicional, de cara a las nuevas medidas adoptadas. 
la experiencia de los estudiantes en dicha coyuntura, intentando abordar, desde el entramado básico del modelo DBO, además de la experiencia individual que contextualizó la decisión de cambiarse de colegio (o bien de permanecer en el establecimiento de origen), algunos elementos contextuales provistos por los colegios emblemáticos.

Con este fin, se realizaron 22 entrevistas a estudiantes de colegios emblemáticos de $3^{\circ}$ y $4^{\circ}$ medio que cursaban esos niveles entre 2013 y 2014: 11 de un colegio emblemático femenino y 11 de un establecimiento similar masculino ${ }^{24}$, ambos de la Región Metropolitana. Se realizaron además tres entrevistas a directivos (directores, orientadores, jefes de UTP, etc.) en cada uno de los establecimientos, lo cual fue además complementado con una revisión de prensa y la correspondiente revisión de documentos, cuando hubo oportunidad de ello. Adicionalmente, se realizó un Focus Group con estudiantes, lo cual relevó principalmente la dimensión colectiva del proceso.

Sobre el material seleccionado, se realizó un análisis de contenido que como categorías de codificación iniciales reconocía las contempladas por el modelo DBO; no obstante, consideraba también la indispensable apertura a la generación de nuevos códigos conforme se fuese requiriendo. Dicha labor fue facilitada por la utilización del software ATLAS ti 8.0.

Todas las entrevistas y el Focus Group fueron codificados y analizados como unidad, circunstancia en la que, si bien se pretende relevar los elementos comunes en la experiencia de los distintos

24 La variable "género" fue considerada desde el diseño original del estudio. La forma en la que esto se implementa es considerando al interior de la muestra de los colegios emblemáticos de la Región Metropolitana uno femenino y otro masculino. Lo anterior es resultado de que los colegios públicos tradicionalmente emblemáticos aún utilizan la separación por sexo de su estudiantado para organizar su labor, circunstancia en la que incluir la variable, además de pertinente, no resultaba complejo. Por tratarse de un estudio compuesto de casos específicos y de naturaleza más cualitativa, no se puede ser concluyente respecto de la existencia de diferencias relevantes entre géneros; no obstante lo cual, tras el correspondiente análisis, lo que prima es una sintonía básica de toma de decisiones orientadas por una racionalidad instrumental, en una coyuntura crítica, en un contexto de información poco clara, con premuras de tiempo y tensionadas por elementos emocionales y simbólicos. 
estudiantes, no siempre se observa esta posibilidad, lo cual no resulta difícil de entender si pensamos que, entre otras cosas y enfrentados a la disyuntiva, algunos decidieron quedarse y otros irse.

\section{5) ¿Qué significaba quedarse y qué significaba irse?}

(D) Deseos

En el análisis desarrollado, la categoría Deseos queda expresada en una combinación de anhelos y expectativas asociados al potencial de movilidad social y económico contenido en la educación en sus distintos niveles.

Cuando estaba en este proceso de paso a la media, mi papá estaba muy preocupado de mi ingreso a la universidad, como que él se proyectaba mucho con el tema de mi futuro (... $)^{25}$ entonces, veamos qué hacemos, porque tampoco tenemos la plata, porque yo no vengo de una familia adinerada, y ahí él, entre contactos, me dice que podían meterme a un liceo emblemático. (VP, Colegio Femenino. Migró)

Se observa que los deseos articulados en torno a anhelos son de los elementos menos heterogéneos de los revisados en el análisis, pues, si bien hay matices, en el contexto de los establecimientos emblemáticos prima la expectativa de ser profesional...

... pero mis expectativas yo creo que iban más de antes en mí que quería ser estudiante universitaria. Y en mi familia igual como que somos la primera generación de universitarios. Todos los demás era como colegios técnicos o técnico profesional. (PAC, Colegio Femenino. No migró)

\section{... de una carrera tradicional}

es que si hubiera dicho "quiero ser bibliotecólogo" me hubieran mirado muy raro. O si realmente quiero hacer otra cosa, algo que no sea una carrera tradicional, hiperconocida, una carrera universitaria,

25 [...] Elipsis utilizada para abreviar, anonimatizar y contextualizar los apartados literales, cuando corresponda. 
me hubieran mirado raro y me lo hubieran preguntado durante un mes entero diciéndome: ien serio quieres eso? (DB, Colegio Masculino. Migró)

\section{...de una universidad pública}

Es que igual en el [nombre de colegio] tenís este tema, si entrevistas a otra gente te va a decir igual, de que es muy enfocado en la universidad. [...] Y es como si, universidad, universidad, universidad. (DB, Colegio Masculino. Migró)

Ya estando adentro empecé a entender más qué era ese colegio, qué oportunidades me daba ese colegio, qué tenía que hacer al ser de ese colegio y ya por primero medio tenía claro que sí o sí quería ir a la universidad. (CF, Colegio Masculino. No migró)

En materia de deseos, observamos que los anhelos y expectativas de los estudiantes de colegios emblemáticos son en general bastante homogéneos y nos remiten a tópicos tradicionales en materia de movilidad social y educación, como son los del futuro y la proyección asociada a este de una carrera universitaria.

\section{(B) Creencias}

Lo expresado en el punto anterior calza con la fuerte relación, para los estudiantes entrevistados, entre educación y movilidad social. Esto determina que la inicial adscripción a los colegios emblemáticos fuese entendida, desde sus posiciones socioeconómicas de origen, como una buena oportunidad para ella, amparada incluso en el relato familiar.

Es la única opción que tengo para sobrevivir, porque si no terminaría peor de lo que estaba antes. Porque yo vivía en una población cuando era chica y me cambié a un barrio mejor, pero si no estudiara, volvería a esa población, porque no tendría cómo salir del hoyo. (BC, Colegio Femenino. No migró)

Mis dos papás venían de familias súper humildes y ellos tuvieron la suerte de estudiar en la universidad y gracias a eso pudieron salir 
adelante. Y veía, muchas veces, mi mamá me ha contado, cómo sus vecinos se fueron quedando atrás. (VC, Colegio Femenino. No migró)

Al parecer, sería la específica conjunción entre Deseos y Creencias aquello que explica la fuerte sensación de amenaza vivenciada por los estudiantes de cara a los cambios en el sistema de admisión.

Y ahí ya fue catastrófico. (...) los que queríamos sí o sí entrar a una estatal, por los temas de aranceles por las becas, qué se yo, y por el prestigio, lo vimos como una amenaza. De verdad fue una [cuestión] que nos dio mucho miedo a todos. (CF, Colegio Masculino. No migró)

Así visto, el cambio se consolida como creencia de una inminente amenaza, en una coyuntura crítica en la que hay incertidumbre y confusión, además de falta de información precisa respecto a cuánto y cómo iban a cambiar las cosas:

en el colegio tampoco sabian bien de que se trataba. (Focus Group, Colegio Femenino)

... mi papá me dijo el año anterior: “-oye, sabís' que van a implementar esta cosa del ranking, quizás te va a convenir cambiarte después. Y yo me negué poh', yo en tercero medio pensaba en egresar del colegio, [...] veía que mis notas no eran las mejores, pero pensaba que mis notas igual me iban a alcanzar, pensaba yo. Y bueno, eso fue ese año. Y ya al otro año, fueron conversaciones más serias: "de verdad te va a convenir...". (LP, Colegio Masculino. Migró)

La cosa es que nadie nos avisó, [había un] un papelógrafo. Lo fuimos a buscar y fue como: ¿chiquillas...? ¿qué es esto? ¿Si tengo 5,9, por qué tengo 590? y ¿Por qué las chiquillas que estaban más arriba tenían como 700? Y eso fue miedo. Fue como oooooh. igual poco! (Focus Group, Colegio Femenino)

De esta forma, cuando el sistema se empieza a implementar, la incredulidad inicial es reemplazada por desazón y miedo. 
... yo me empecé a cuestionar mucho, como si hubiese seguido en mi colegio anterior [previo al emblemático], que era mucho más básica la educación, hubiese tenido un promedio mucho mayor, me hubiese beneficiado mucho más el ranking. (...) A nosotros como que nos dio como terror. (PAC, Colegio Femenino. No migró)

Y, naturalmente, el cambio de expectativas de cara a los nuevos mecanismos que se implementan...

Y así una misma empezaba a bajar sus expectativas, porque al principio todas queríamos las universidades estatales (...) Ese era el sueño de nuestros papás (...). Y eso también contribuyó al pánico, porque eran las estatales las que estaban con esas cantidades de ranking, y uno se empezó a dar cuenta de que ya no voy a entrar (...). Entonces ahi vimos: "chiquillas, tenemos estas universidades privadas que también son buenas". (Focus Group, Colegio Femenino)

Y fue como, "oh vaya". Con esto empecé a sacar cálculo, incluso sí, saqué cálculo, vi que esta cosa me dejaba enfermo, tipo esta cosa, ohhh con suerte a la $P$, la A [nombres de universidades privadas] y qué paja, no quiero esto (...). (DB, Colegio Masculino. Migró)

... como también la estructuración de una fuerte resistencia, al menos en un inicio...

Como que se empezó a notar el ambiente, como que algo estaba pasando y que iba a tener consecuencias. (CC, Colegio Femenino. Migró)

... en el año 2014 (...) hubo movilización, marchas, paros masivos, en todos los liceos. (Focus Group, Colegio Femenino)

... para posteriormente, y una vez decepcionados de la impotencia de únicamente expresar su malestar y lo infructuoso de protestar en torno a esto, comenzar a barajar otras acciones:

En verdad fue eso. Fue (...) pa' todos lados. Agitar muchas partes, y como vieron que ya no estaba funcionando, empezaron a irse del colegio. (CF, Colegio Masculino. No migró) 
... se generó primero como una preocupación general, que evolucionó después a una toma de decisiones por parte de nosotros. (FA, Colegio Masculino. Migró)

Más allá de los comprensibles componentes emotivos, es importante destacar en primer lugar, a partir del análisis, la creencia compartida en la educación tradicional como plataforma a la educación universitaria y el potencial de movilidad social asociado como línea base de las creencias de este tipo de estudiantes. Asimismo, en segundo término, la posterior internalización de la creencia que, producto del cambio, ya no se podía sostener las mismas expectativas, circunstancia en la cual había que hacer algo. Es la urgencia frente a esta necesidad de "hacer algo" lo que configura el punto de inflexión respecto de que hay que tomar decisiones.

Y en un momento un amigo mío, sin decirle a nadie, se cambió, desapareció. (...) Y todos quedamos como ṕadentro. Esto se viene en serio. (LP, Colegio Masculino. Migró)

(O) Oportunidades

Frente a la necesidad de encontrar alternativas de acción se encuentra el resquicio procedimental, el que, una vez identificado, se consolida en una imprevista (al menos para la mayoría originalmente) oportunidad.

Entonces es cuando todos cacharon la pillería de cambiarse. (VC, Colegio Femenino. No migró)

No obstante, la identificación de un mecanismo viable para hacer frente a esta situación no está exento de incertidumbre y disyuntivas dilemáticas frente a una eventual decisión:

"Soy sincera, estoy aprovechándome de esta situación, pues hecha la ley, hecha la trampa". (Cita extraída de revisión de prensa en Galarce, 2014)

Nosotras también dijimos "chuta, ¿qué hacemos nosotras? Hay gente cambiándose. ¿Lo hacemos? ¿No lo hacemos?”. (Focus Group, Colegio Femenino) 
... se puso una especie de tensión dentro del mismo colegio. ¿Qué significaba quedarse y qué significaba irse? (DB, Colegio Masculino. Migró)

\section{(A) Acciones y decisiones}

En lo esencial, aquello que permite dilucidar esta instancia dilemática es la identificación de cuál sería, en general, el objetivo de incurrir en tal maniobra:

... el gran objetivo que se plantearon algunos, entrar a un colegio que les permitiera, digamos, obteniendo notas buenas, tener un ranking lo más alto posible. (FA, Colegio Masculino. Migró)

Y, en torno a la eventual consecución de ese objetivo, proceder a evaluar la maniobra migratoria en términos de costos y beneficios:

La cosa es que llegó un punto donde todo el mundo estaba con la calculadora todo el tiempo, sacando como para clasificar al mundial, pero en este caso para ir a la Chile. Era como: "pucha si ranking tata-ta y si saco 750 en esto y esto", y es como funciona y empezaba a calcular cosas. (DB, Colegio Masculino. Migró)

Mi pensamiento fue: ¿Para qué estar en un colegio que te prepara tanto para la PSU, si al final la enseñanza no vale nada, porque afecta mucho el ranking? En ese caso, me aseguro con un buen ranking en un buen colegio, teniendo mi mismo método de estudio, que ya era exigente, y me va a ir mejor. Al final, voy a tener mejores notas en un colegio mediocre, por así decirlo. (MM, Colegio Femenino. Migró)

Lo que hizo irme, llegar al otro colegio, fue el tema del ranking, ver que mi puntaje ranking subía más de 100 puntos y me abría muchas más posibilidades de quedar en una universidad estatal, en la Chile o en la Usach, que eran las que yo quería. Y en caso de quedar, optar a becas. (LP, Colegio Masculino. Migró)

La importancia del grupo de pares en el colegio de origen también es "puesta en la balanza" al momento de decidir. No obstante, en algunos casos resulta insuficiente para objetar la conveniencia de la maniobra. 
... fue como una decisión, averigüé los costos, los beneficios, en verdad no era mucho lo que sacrificaba, o sea las relaciones sociales como los lazos que tenía con compañeros, la graduación en el colegio también. Pero cuando saqué el cálculo de cuánto me beneficiaba estar en ese colegio dije: "Ah, mejor me cambio". (DA, Colegio Masculino. Migró)

... principalmente mi grupo de amigos cercanos, [y] por lo que significa el colegio para mí, me daba mucha pena irme. Eran los puntos que me hacian quedarme. Pero para el tema de entrar a la universidad, (...) claramente con las notas que tenía en ese entonces no me iba a alcanzar por ranking (...). Eso, sumado a que muchos de mis compañeros también migraron, me hizo tomar la decisión de salir yo también del colegio. (FA, Colegio Masculino. Migró)

Conforme a la cita anterior, es posible observar que el accionar de los pares de alguna manera genera confianza para la ejecución de la maniobra. No obstante, y a pesar del accionar de varios estudiantes, el resultado de la elección no siempre fue el de irse, pues otras percepciones se desarrollan en el proceso.

... me dio miedo cambiarme, como que no quería, como que apechugué en ese momento con lo que me tocara. O sea, lo pensé solamente. (...) pero yo no me atreví porque sabía que mi liceo era bueno. Y no tenía plata igual para costear, porque yo nunca he pagado nada. (PAC, Colegio Femenino. No migró)

Yo estaba bien desmoralizado en ese momento y tampoco me lo planteé. Porque me sumí un poco en la pena, de haber estado en ese colegio con tanta gente y ahora estaba solo. (FA, Colegio Masculino. No migró)

... tenía como una ambivalencia con el liceo y mis compañeras, pero aun así quería salir de ahí, así que mientras más rápido lo hiciera mejor, y en realidad si me cambiaba no iba a ser tan rápido. No lo recuerdo tampoco, porque siempre tuve esa idea de que yo entraba ahí y salía como sea, a los 4 años a como diera lugar. (PC, Colegio Femenino. No migró)

Más allá del natural miedo e incertidumbre observados en el desconcertante panorama, resulta interesante encontrar no pocos 
casos en los cuales también hay elementos de orden normativo, más que exclusivamente regulativos, capaces de ilustrar la evaluación en torno a costos y beneficios, lo cual incluso es capaz de poner en jaque el efecto del accionar de pares.

Eran de mis mejores amigos en el colegio y se fueron. (...) me dijeron "ya (...) vamos a ir tal día. Lleva los papeles y nos cambiamos todos", y yo les dije "ya"... Y ese día que ellos se fueron a matricular yo los llamé y les dije: "Cabros, no voy a ir. Me quedo. (...) No me parece correcto hacer esta [cuestión]", les dije. Yo dije no, yo no me cambio, y si no entro, no entro no más. (CF, Colegio Masculino. No migró)

Incluso se observan situaciones mixtas, en las que aun la decisión de irse va acompañada de cierta reflexión normativa respecto de cómo sus acciones podrían afectar a otros.

Yo no quería llegar a un entorno a aprovecharme de los chicos que ya estaban ahi y habian formado su vida. Y yo, llegar de la nada, a quitarles su puesto, a quitarles su lugar, no. Entonces me fui a un lugar donde mi ranking no subía. 40 puntos no es nada. Entonces yo no le iba a quitar el puesto a nadie. Yo iba solamente porque quería irme no más. (CN, Colegio Masculino. Migró)

Pese a que hay instancias de un accionar orientado por cierta norma ética, es posible observar que el interés por optar a las universidades tradicionales es majadero y persistente, y por ello buscan nuevas oportunidades:

... hablando con mis compañeros vi la posibilidad de que podíamos volver a tener como una oportunidad, se podría decir, si es que utilizábamos la vía del ranking, básicamente que había que hacer el cambio (...). No sé. Yo ya estaba como con la idea de que iba a entrar a una privada. Igual intentemos la posibilidad de que pueda entrar a una estatal. Así que me cambié. (VL, Colegio Masculino. Migró)

Interesante también la situación en la cual, no habiendo un panorama muy nítido, alguien es capaz de decidir partir, para no arrepentirse en el futuro de no haber aprovechado la oportunidad: 
Quizás, más que todo era la oportunidad. Tu veís' una oportunidad y después diría “¿Por qué no la tomé?". Entonces, ante la duda, mejor la tomé. (IS, Colegio Masculino. Migró)

Lo que sí parece primar, en general, es la reflexión en torno al interés individual.

... para quedar en el ranking, en lo más arriba del ranking (...) implicaba que ibas a desplazar a otra persona que ya tenía años ahí. Y yo veía y les explicaba que, la verdad, con eso los colegios estaban aumentando la matrícula, estaban viendo, pero no estaban resguardando a sus alumnos. (...) No digamos que eso fue una situación que ellas consideraban, no, porque ellas ya tenían la decisión. (Orientadora, Colegio Femenino)

\section{Información}

En este contexto de incertidumbre y toma de decisiones, la gradualidad y posterior aceleración de la migración encuentra explicación en la progresividad del proceso de captación, procesamiento y difusión de información respecto de las alternativas disponibles:

Fue gradual. No fue que de un día para otro se fueron todos. De hecho, partió uno y después se fueron dando el dato de los colegios. (CJ, Colegio Masculino. No migró)

... estaba tan en pánico con la situación, que hice una búsqueda, pero exhaustiva, porque habia unos programas donde ver los rankings de colegios, y yo empecé, todos los colegios de Puente Alto yo empecé a preguntarles. (Focus Group, Colegio Femenino)

yo divulgué esa lista en mi grupo de amigos, y la divulgué con otros chicos de otros cursos y con amigos de otros colegios que estaban pasando también lo mismo. (...) y como que todos: "ooohh, mira los puntajes (...), ¿Cuánto nos subiría esto?' (...) me fue hablando gente después, me decían como que le mandara la lista, que estaban viendo a qué colegios, en dónde quedaban, y les empecé a decir dónde estaban los colegios y se fueron cambiando. (DA, Colegio Masculino. Migró) 
Al respecto, es interesante destacar que, en el flujo migratorio, se observan al menos dos momentos. El primero asociado a la coyuntura crítica y que termina con la saturación y extinción de vacantes en los establecimientos que reciben a los primeros migrados, y un segundo en el cual la maniobra se realiza a colegios menos obvios y de los cuales la información no siempre estaba disponible.

... estuvimos todas buscando colegios y había dos colegios que como que se habia pasado la voz de que estaban recibiendo gente y que tenian rankings bajos. Entonces, todas queríamos irnos ahí, pero los colegios estaban saturados, entonces no iban a recibir más gente. (Focus Group, Colegio Femenino)

hubo otros colegios y eso fue bastante raro, que fueron de primera ola de migración, que eran el [colegio público no emblemático], estos como de primera ola de migración las primeras dos semanas, que se llenaron de gente del [nombre de un colegio emblemático femenino] $y$ del [nombre de un colegio emblemático masculino] y que por esa misma razón ya no eran una opción para migrar, porque al final por así decirlo (...) quedaba con los mismos. Al final voy a subir dos puntos (...) y ahí me fui al [otro] de Recoleta. (DB, Colegio Masculino. Migró)

"iChiquillas encontré este colegio!”, y de repente me encontré con esta página Liceo (...) de La Florida y ahí si poh'. (Focus Group, Colegio Femenino)

Fue así como, por ejemplo, la improbable ejecución de la maniobra migratoria a establecimientos invisibles, al menos desde la óptica que ilustra lo emblemático, generó situaciones de negociación muy interesantes e impensadas al momento de iniciar este estudio. Un ejemplo es el detallada en el siguiente apartado, y que pone de manifiesto la fragmentación existente en el sistema escolar chileno:

... cuando llegamos, ellos dijeron ya, OK, pero ipor qué se quieren cambiar a estas alturas del año? Vienen de un buen liceo, ¿qué está pasando? O sea, si nosotras estábamos recién enterándonos, imagínense ellos, que sus alumnos no estaban ni ahí con la PSU, no tenían idea de lo que estaba pasando. Y al principio les explicamos 
y nos dijeron "sí vénganse, vénganse", y después nos dijeron que no, que no nos iban a recibir en el liceo. (...). Es que (...) habia un niño que era como el mateo, que tenía como promedio 6,1 y esa directora, cuando fuimos a consultar en primera instancia no sabia, después se informó y nos dijo que no, y nosotras, "ipero por qué?". Y lo que pasa es que si ustedes vienen con promedios más altos afectan la beca de excelencia académica, que era lo más importante, la beca de excelencia académica, ella estaba muy al tanto, y afectan a mi niño porque llegan con promedio y se la quitan. Y mi papá dijo: "deje entrar de 5,9 para abajo", y yo tenía 5,9-5,7, y ese fue el requisito para entrar. Y después llegaron los del [otro colegio emblemático] y todo era de 5,9 para abajo, nadie con 6,0. (Focus Group, Colegio Femenino)

\section{Conclusión}

Abriendo una reflexión final, a manera de conclusión, es posible observar que lo interesante del caso, y con cuya reflexión puede aportar la sociología a las políticas públicas, es que la implementación de una medida que, en términos de su construcción, apuntaba a remediar una situación de inequidad (conforme a la intención declarada por el CRUCH), respondiendo a la demanda que en años previos había realizado el movimiento estudiantil, liderado muchas veces por estudiantes de estos mismos colegios, determina la activación —al parecer inesperada — de mecanismos individuales de acción que, de alguna forma, encuentran resquicios para obtener réditos (hasta el último momento) del dispar background educativo que, en términos de logro académico, poseían los estudiantes de los establecimientos emblemáticos.

Ya en 2013 Larrocau, Ríos y Mizala (2013) señalaban lo importante que era explorar la forma en la que los estudiantes deciden sus preferencias por carreras y universidades, en tanto éstas “... siguen patrones muy complejos de selección, que responden a intereses propios y no a una lógica predecible por el Proceso de Admisión" (p. 99). Muy en sintonía con esta recomendación, es posible pensar que el proceso de toma de decisiones asociado a la migración entre colegios puede ser abordado como una respuesta orientada por los intereses individuales de los estudiantes, frente a la modificación 
resultante de la incorporación del ranking y que habría afectado tanto a los estudiantes de educación secundaria como a sus colegios.

Desde cierto punto de vista, la acción de cambio antes referida se comprendería eminentemente a partir de un accionar que opera como respuesta a un cambio regulativo, el que, no obstante, también podría traer aparejados importantes efectos en un nivel normativo, pues la maniobra migratoria no parece haber estado exenta de ciertos cuestionamientos éticos y valóricos. En este sentido, es posible afirmar que hay una racionalidad estratégica en el proceso por parte de los estudiantes, aunque naturalmente no todo se reduce a ello. Hay reacciones de orden moral y normativo capaces de ilustrar el referido proceso, en el entendido de que varios de los estudiantes que se quedaron (e incluso algunos de los que finalmente partieron) también debieron procesar esos elementos en su decisión.

Al respecto, y tras lo revisado, podemos coincidir con Ball (1994) cuando indica que las políticas son simples y crudas, mientras que las prácticas con las que los actores se apropian de ellas son sofisticadas, contingentes, complejas e inestables, a lo cual podríamos agregar "dotadas de racionalidad", aunque no necesaria ni exclusivamente de una racionalidad economicista estricta. No obstante, la aprensión representada por Gil, Paredes y Sánchez (2013), en el sentido de que considerar para el ranking la posición relativa del estudiante respecto a su curso o generación podría inducir una indeseada dinámica de competencia, conforme a lo revisado, resulta atendible. Respecto de esto, consultado un directivo por esta situación, realiza una interesante reflexión, indicando:

Normalmente las personas tienden (...) a manejar los entornos. Es una cuestión de supervivencia. Si a uno le colocan una traba o le colocan un obstáculo tal, lo que tratan de hacer las personas es: "cómo yo modifico mis conductas o mis insumos con tal de manejar esa nueva situación, para poder salvar los requerimientos que de alguna forma el sistema me está imponiendo". (Directivo, Colegio Masculino)

Precisamente, desde esta perspectiva, el modelo DBO releva ciertos elementos para el análisis aquí realizado, en tanto prescinde 
de enjuiciar valorativamente el accionar de los individuos y modela, a partir de lo declarado, la forma en la que el fenómeno se manifestó y cómo se procesó y se vivenció por parte de ellos.

Cuando se produce esto y perciben que los va a afectar individualmente, bueno, el "sálvese quien pueda" los lleva a tomar decisiones de cómo salir rápidamente (...) e irse a otro establecimiento educacional que tuviese niveles de exigencia menores. Y por las notas NEM que ellos tenían (...), ellos iban a quedar inmediatamente por sobre el diez por ciento de la generación donde fueron. (Directivo, Colegio Masculino)

Consultado sobre esto, el experto en educación José Joaquín Brunner (en Galarce, 2014 b), al indicar que en el fondo quienes migran estarían tratando de sobreasegurarse, señala:

Pero no se puede juzgar para nada. Están aprovechando un hueco, una oportunidad que el sistema de ranking abre a algunos alumnos. Tampoco es algo que puedan hacer muchos. Es un grupo pequeño que está en colegios de alto rendimiento académico. Yo no haría un juicio negativo de estos jóvenes...

Contextualizando la situación, es posible agregar ciertas implicancias materiales de ésta, las que en ese momento aún constituían una incógnita.

Era como asegurar ese poco porcentaje para lo que ella pensaba en la educación superior. Acuérdese que en ese minuto tampoco estaba la gratuidad. Entonces o era la beca o era el crédito CAE. (UTP, Colegio Femenino)

En ese contexto, la inserción de los jóvenes decisores en la red de las distintas políticas públicas en materia de educación superior (tránsito, acceso, financiamiento, etc.) parece detonar ansiedades en torno a ciertos elementos de sus proyectos de vida, las que, a su vez, activan ciertos dispositivos de racionalidad estratégica orientados a sortear la coyuntura que les presenta la contingente configuración institucional. De alguna forma, esto también desactiva el ánimo de implicarse en proyectos de acción colectiva orientados a promover la reforma de la inequidad estructural del sistema educativo; acción 
colectiva de la que, en años previos, los estudiantes de los liceos emblemáticos habían sido protagonistas privilegiados.

Que muchos estudiantes hayan pensado en retirarse del colegio tiene que ver con el fenómeno de la individuación, donde lamentablemente las generaciones actuales son demasiado individuales. Prácticamente piensan en cómo las cosas les afectan a ellos de forma individual, más que como un colectivo social. (Directivo, Liceo Masculino)

En cuanto a lo estrictamente teórico, y conforme a lo revisado, es posible observar que el modelo DBO permite arrojar luces acerca de la situación, principalmente de la dinámica regulativa del proceso de implementación. Respecto de este tipo de dinámicas y, en específico, de la toma de decisiones asociadas a sistemas educativos, Gambetta (1987) planteaba la disyunción de entender si las acciones desarrolladas por los estudiantes debían ser entendidas como que si estos saltaban o eran empujados ${ }^{26}$. Hay ahí, quizás, un falso dilema, en tanto uno podría entender, también figurativamente y citando al refranero, que "cuando una puerta se cierra, una ventana se abre". No a todas las personas les agrada de la misma forma saltar por ellas mismas, lo cual proyecta al ámbito de lo normativo lo que ha sido solo incidentalmente abordado en el presente trabajo.

$\mathrm{Al}$ respecto, es posible observar que, junto con lo normativo, también hay otros elementos que exceden el foco estricto del presente trabajo y nos proyectan de manera más sólida a interrogantes tales como: ¿de qué forma participan (o no) los padres en la decisión? ¿Cuál es el rol de profesores y directivos de los establecimientos? Y, probablemente la más interesante de todas, ¿de qué forma los estudiantes de ese entonces evalúan con el tiempo la situación enfrentada y la decisión tomada? (evaluación ex post).

De ninguna manera el análisis DBO parece agotar la complejidad de la situación. No olvidemos que es solo un modelo que, aplicado analíticamente, es capaz de ilustrar un fenómeno, en tanto ilumina algunos aspectos, mientras omite otros.

26 Where they pushed or did they Jump? 
En ese sentido, es posible señalar que del terreno indagado emergen una serie de otras dimensiones, entre las que cabe destacar la dimensión simbólica y cognitiva de aquello que es connotado como lo "emblemático". Desde esta perspectiva, es posible abordar cómo el imaginario de los establecimientos emblemáticos se tensiona y fractura en un proceso crítico. Valga lo anterior para relevar el hecho de que el levantamiento realizado a propósito de este complejo caso está compuesto de diversos aspectos que van más allá del modelo DBO y que son capaces de complementarlo y enriquecerlo, entre los cuales destaca, por ejemplo, el tema de las emociones implicadas en el proceso y la distinción por género de ellas. Al respecto, de momento, el procesamiento de información no obtiene nada conclusivo, pero sí ciertos indicios para profundizar en ello:

Llegué y todas hablaban de cuándo se iba una o la otra. Era un ambiente deprimente, profesoras llorando, niñas llorando. Fue duro para todas. Pero ese ambiente me ayudó a tomar la decisión. (Cita extraída de revisión de prensa) ${ }^{27}$

Nosotros no teníamos ese lujo; porque claro, éramos hombres. No podíamos llorar. (FA, Colegio Masculino. No migró)

Valga lo anterior para relevar uno de los atractivos de un modelo como el DBO y que es que, en su carácter modular, permite ser complementado en su análisis en tanto pueda ser formalizado, tal como lo hace Elster (1999). Lo anterior cobra especial relevancia como corolario si atendemos a lo expresado por una directiva del Colegio Femenino:

Creo que la ansiedad, la incertidumbre, la angustia de saber que "tengo una ganancia”, las llevó a buscar a irse a los colegios periféricos de Santiago. Lo hicieron obviamente en un minuto donde ellas tenían asegurada una preparación, entonces yo creo que era una herramienta, más que pensar en la PSU. (UTP, Colegio Femenino)

27 Galarce, Arturo. Las Últimas Noticias; 08 de agosto de 2014. "Carmela Carvajal sufre éxodo de alumnas que buscan mejorar su puntaje PSU". 
A manera de cierre, resulta imprescindible recordar la idea de que, más allá de lo exclusivamente regulativo, y respecto de lo cual el modelo DBO puede arrojar luces, se trata esta de una investigación acerca de la forma en la que los destinatarios de las políticas públicas en educación superior procesan su recepción (Gornitzka, Kogan y Amaral, 2005) por medio de interacciones. Esto es distinto de aquellas investigaciones que, en materia de políticas públicas, abordan aspectos más propios de lo político, tales como identificar qué problemas deben ser abordados por medio de determinadas políticas y qué se debe hacer al respecto (Scharpf, 1997). En este contexto, lo regulativo y su asociación a algunos componentes cognitivos parecen ser indispensables, más nunca suficientes, en tanto la complejidad de estos procesos también parece demandar la incorporación de lo simbólico y, tratándose de estudiantes secundarios y adolescentes, de lo emotivo.

\section{Referencias}

Aguiar, F., De Francisco A. y Noguera J. A. (2009). Por un giro analítico en sociología. Revista Internacional de Sociología (RIS), 67(2), 437-456.

Babbie, E. R. (2013). The basics of social research. Cengage Learning.

Ball, S. J. (1994). Education Reform: A Critical and Post-structural Approach, Chapter 3. Buckingham: Open University Press.

Barbera Filippo, A. (2006). Star is Born? The Authors, Principles and Objectives of Analytical Sociology. Papers, 80, 31-50. Universitat Autònoma de Barcelona. Departament de Sociologia.

Bernstein, B. (1993). La Estructura del Discurso Pedagógico. Madrid: Paideia.

Bornmann, L. (2010). Die analytische Soziologie: Soziale Mechanismen, DBOTheorie und Agentenbasierte Modelle. Österreichische Zeitschrift für Soziologie, 35(4), 25-44.

Boudon, R. (1974). Education, Opportunity, and Social Inequality: Changing Prospects in Western Society. New York: Wiley-Interscience.

Braun, A., Ball, S. J., Maguire, M. \& Hoskins, K. (2011). Taking context seriously: Towards explaining policy enactments in the secondary school. Discourse: Studies in the cultural politics of education, 32(4), 585-596. 
Carrasco, E., Zúñiga, C. y Espinoza, J. (2014). Elección de carrera en estudiantes de nivel socioeconómico bajo de universidades chilenas altamente selectivas. Calidad en la educación, (40), 95-128.

Coleman, J. (1966) Equality of Educational Opportunity. (COLEMAN) Study (EEOS).

Dunning, T. (2012). Natural experiments in the social sciences: a design-based approach. Cambridge University Press.

Elster, J. (1976). Boudon, education and the theory of games. Social Science Information, 15(4-5), 733-740.

Elster, J. (1999). Alchemies of the Mind: Rationality and the emotions. Cambridge University Press.

Enders, J., Jeliazkova, M., McGuiness Jr., A. \& Massen, P. (2003). Higher Education Policy Formulation and Implementation: A Framework for Case Análisis. Prepraded for the CHER $16^{\text {th }}$ annual conference. "Reform and Change in Higher Education: Renewed expectations and improved performance?"

Galarce, Arturo a). Las Últimas Noticias; 08 de agosto de 2014. "Carmela Carvajal sufre éxodo de alumnas que buscan mejorar su puntaje PSU".

Galarce, Arturo b) Las Últimas Noticias; 08 de agosto de 2014. "En el fondo están tratando de sobreasegurarse".

Gambetta, D. (1987). Were they pushed or did they jump? Individual decision mechanisms in education. Cambridge University Press.

Gamoran, A. \& Long, D. A. (2006). Equality of Educational Opportunity: A 40-Year Retrospective. WCER Working Paper No 2006-9, December.

Gil, F., Paredes, R. \& Sánchez, I. (2013). El ranking de las notas: inclusión con excelencia. Temas de La Agenda Pública. Centro de Políticas Públicas UC, 8(60), 3-19.

Goldthorpe, J. H. (1998). Rational Action Theory for Sociology. The British Journal of Sociology, 49(2), 167-192.

Gornitzka, A., Kogan, M. \& Amaral, A. (eds.). (2005). Reform and Change in Higher Education. Analyzsing Policy implementation. Springer.

Hedström, P. (2005). Dissecting the social: On the principles of analytical sociology. Cambridge: Cambridge University Press.

Hedström, P. \& Udehn, L. (2011). Analytical sociology and Theories of the Middle Range, in The Oxford Handbook of Analytical Sociology. USA: Oxford University Press. 
Hedström, P. \& Bearman, P. (2011) What is Analytical Sociology all about? An Introductory Essay in The Oxford Handbook of Analytical Sociology. USA: Oxford University Press.

Kaidesoja, T. (2012). The DBO theory of action and distributed cognition. Social Science Information, 51(3), 311-337.

Kain, J. F., Singleton, K. (1996). Equality of educational opportunity revisited - Special Issue: Earnings Inequality. New England Economic Review.

Larroucau, T., Ríos, I. y Mizala, A. (2013). Efecto de la incorporación del Ranking de Notas en la selección universitaria. Reporte técnico. Santiago de Chile: Departamento de Evaluación y Registro Educacional (DEMRE). Anexos.

Leyton, D. (2015). La transición de la escuela a la educación superior en estudiantes provenientes de contextos vulnerados. Santiago de Chile: Universidad Alberto Hurtado.

Leyton, D., Vásquez, A. y Fuenzalida, V. (2012). La experiencia de estudiantes de contextos vulnerables en diferentes Instituciones de Educación Superior Universitaria (IESU): Resultados de investigación. Calidad en la Educación, (37), 61-97.

Manzo, G. (2010). Analytical Sociology and Its Critics. Archives Européennes de Sociologie, 51(1), 129-170.

Manzo, G. (2011) Educational choices and educational traps. Towards an integration between computational and statistical modelling in the sociology of social stratification. $N^{\circ}$ 1. GEMASS Working Papers, GeWop.

Manzo, G. (2013). Educational choices and social interactions: a formal model and a computational test. Comparative Social Research, 30, 47-100.

Noguera, J. A. (2006). Introduction: why we need an analytical sociological theory. Papers: Revista de Sociología, 07-28.

Noguera, J. A. (2003) ¿Quién teme al individualismo metodológico? Un análisis de sus implicaciones para la teoría social. Papers, 69, 101132. Universitat Autònoma de Barcelona. Departament de Sociologia.

Paulus, N., Geoffroy, E. y Mendoza, M. (2010). El tránsito a la vida post secundaria: la experiencia de la primera generación de egresados de un liceo de una zona popular en Santiago. RIEE. Revista Iberoamericana de Evaluación Educativa, 3(2), 182-199.

Sabatier, P. (2005). From Policy Implementation to Policy Change: A Personal Odyssey, in Reform and Change in Higher Education, Analysing Policy Implementation. Springer. 
Scharpf, F. W. (1997). Games Real Actors Play. Actor-Centered Institutionalism in Policy Research. Theoretical Lenses on Public Policy. USA-UK: Westview Press.

Sin, C. (2014). The policy object: A different perspective on policy enactment in higher education. Higher Education, 68(3), 435-448.

Scott, W. R. (1995). Institutions and Organizations. California: Sage Publications.

Treviño, E., Scheele, J. \& Flores, S. M. (2014). Beyond the Test Score A Mixed Methods Analysis of a College Access Intervention in Chile. Journal of Mixed Methods Research, 8(3), 255-265

Troiano, H. (1999). El procés de reforma dels plans d'estudis de les titulacions universitàries d'informàtica. Estudi de casos des d'una perspectiva sociologica. Tesi doctoral presentada en el Departament de Sociologia de la Universitat Autònoma de Barcelona. Director de tesi: Josep M. Masjuan.

Trowler, P. (2003). Education policy. Routledge.

Trowler, P. R. (Ed.). (2002). Higher Education Policy and Institutional Change. Intentions and Outcomes in Turbulent Environments. London: Society for Research into Higher Education and Open University Press.

Witte, J. (2003). A theoretical framework for an international comparative study of the introduction of two-tier study structures in the context of the Bologna process. Paper submission to the CHER Conference, 4-6 Septembre 2003. Center for Higher Education.

Yin, R. K. (2017). Case study research and applications: Design and methods. Sage Publications.

Recibido: 03/06/2019

Aceptado: 29/01/2020 